\title{
Ornstein-Zernike Decay in the Ground State of the Quantum Ising Model in a Strong Transverse Field
}

\author{
Tom Kennedy \\ Department of Mathematics, University of Arizona, Tucson, AZ 85721, USA
}

Received October 8, 1990; in revised form December 3, 1990

\begin{abstract}
We consider the quantum mechanical Ising ferromagnet in a strong transverse magnetic field in any number of dimensions, $d$. We prove that in the ground state the power law correction to the exponential decay of the two point function is $d / 2$. The proof begins by writing the ground state as a classical system in one more dimension. (Thus the classical Ornstein-Zernike power of $(d-1) / 2$ becomes $d / 2$ ). We then develop a convergent polymer expansion and use the techniques of Bricmont and Fröhlich [5].
\end{abstract}

When a lattice spin system is away from any critical points, the truncated correlation functions usually decay exponentially. This exponential decay is typically accompanied by a power law correction, i.e., the decay goes as $\exp (-|x| / \xi) /|x|^{p}$. An interesting question is to determine this power $p$. For classical systems the "generic" power is $(d-1) / 2$. This is known as Ornstein-Zernike decay and has been proved in a variety of models by a variety of methods. (The literature in the classical case is vast. Some of the early references may be found in [5].) Now consider the ground state of a quantum mechanical spin system that is not critical. (For example, take the quantum mechanical Ising model in a strong transverse magnetic field.) This ground state is like a classical system in one more dimension, so the Ornstein-Zernike decay would be a power of $d / 2$.

We prove that the power is indeed $d / 2$ for one of the two point functions in the quantum mechanical Ising model in a strong magnetic field. The Hamiltonian of this model is

$$
H=\sum_{j}\left(1-\sigma_{j}^{z}\right)-\varepsilon \sum_{\langle i j\rangle} \sigma_{i}^{x} \sigma_{j}^{x}
$$

with $\varepsilon$ small. When $\varepsilon$ is sufficiently small this model has been proven to have a unique ground state [18]. We only consider the two point function $\left\langle\sigma_{i}^{x} \sigma_{j}^{x}\right\rangle$.

Our proof begins by using the Trotter product formula to write the ground state of the quantum system as a classical system in one more dimension. An early use of this now standard technique is Ginibre's proof of the existence of long range 
order in the highly anisotropic Heisenberg antiferromagnet at sufficiently low temperatures [10]. It was also used by Thomas and Yin $[24,25]$ to study the low temperature states of various models. Suzuki [23] used this formula to show that the model we study can be expressed as the limit of a sequence of classical Ising models in one more dimension.

The next step in the proof is to develop a convergent expansion for the classical system in one more dimension. This expansion is complicated by the continuous nature of the extra dimension. We approximate this continuous time system by a discrete time one and develop an expansion with the convergence uniform in this approximation. (For a different approach to controlling this approximation in the quantum Ising model in a random transverse field see Campanino et al. [7].) Our expansion is driven by the smallness of the parameter $\varepsilon$. Thus one can think of such an expansion as a rigorous version of perturbation theory. Completely different approachs to making the ground state perturbation theory rigorous have been developed by Kirkwood and Thomas [13] and by Albanese [1]. Neither of these approaches uses the Feynman-Kac formula. Kirkwood and Thomas write the Schrödinger equation in a clever form and use it to develop the expansion. Albanese shows that one can construct a "dressing transformation" which takes the $\varepsilon=0$ ground state into the ground state for nonzero $\varepsilon$. Having developed a convergent expansion for the two point function we then use the techniques of Bricmont and Fröhlich [5] to prove the Ornstein-Zernike power law correction to the exponential decay. Their techniques are similar to techniques used by Gallavotti [9] to study the phase separation line in the two dimensional classical Ising model. The Bricmont Fröhlich method must be modified somewhat for our quantum mechanical model. The modification is similar to the method used by Dobrushin, Kotecky and Shlosman to study the surface tension in the classical Ising model at low temperature [8].

In classical systems the power law correction to the exponential decay is not always $(d-1) / 2$. In Sect. 3 we consider some quantum spin systems in which the power is not $d / 2$. An important question on which we can shed no light is whether any of these counterexamples are generic in the sense that if one adds a small finite range perturbation to the Hamiltonian then the decay is still not of the OrnsteinZernike form.

At first glance it appears that it should be straightforward to prove OrnsteinZernike decay in a large class of quantum systems by the following method. For many models that are small perturbations of systems with a trivial ground state, e.g. the model studied in this paper, one can show that the model is equivalent to a classical model in one more dimension by introducing a "blocking" in the time direction that results from the Trotter product formula. Roughly speaking, one introduces a time scale $\tau$ and divides the time axis $[0, \beta]$ into segments of length $\tau$. Such a blocking technique was used by Thomas and Yin in their study of the quantum mechanical Ising model in a strong transverse magnetic field and the highly anisotropic Heisenberg model at low temperatures [24, 25].

One can then hope to prove Ornstein-Zernike decay in this classical system by one of the many methods developed for the classical case. The problem is that the Hamiltonian of the resulting classical system does not have finite range. It has been asserted that several methods for proving Ornstein-Zernike decay for models with a finite range Hamiltonian can be extended to infinite range Hamiltonians, but this is not correct without some fairly restrictive conditions on the Hamiltonian. A counterexample may be constructed as follows. Kirkwood and Thomas [13] 
showed that when the magnetic field is large enough, the ground state of the model we are considering has a representation as a classical model in the same number of dimensions. The Hamiltonian of this classical model is of the form

$$
H=\sum_{V} c(V) \sigma(V),
$$

where $V$ is summed over all finite sets of sites, $\sigma(V)=\prod_{i \in V} \sigma_{i}$ and $c(V)$ is a real coefficient. The Hamiltonian contains terms with arbitrarily large $V$, but $c(V)$ has exponential decay of the form $|c(V)| \leqq \exp (-m\|V\|)$, where $\|V\|$ is the cardinality of the smallest connected set of sites containing $V$. If such decay were indeed sufficient to imply Ornstein-Zernike decay, then the power for this model would be $(d-1) / 2$, but we will prove it is $d / 2$.

Finally we should comment on whether our results can be extended to the ground states of other quantum spin systems. A natural model to consider next is the anisotropic antiferromagnet

$$
H=\sum_{\langle i j\rangle}\left[\sigma_{i}^{z} \sigma_{j}^{z}+\varepsilon \sigma_{i}^{x} \sigma_{j}^{x}+\varepsilon \sigma_{i}^{y} \sigma_{j}^{y}\right],
$$

where $\varepsilon$ is small. This model has two infinite volume ground states. One can develop a convergent expansion for this model similar to the expansion in this paper, but the geometric analysis of the resulting expansion is more complicated. We have not been able to use it to prove that the power law correction to the exponential decay is $d / 2$. We consider it an open and interesting question to study the power law correction in the ground state of other quantum spin systems.

\section{The Main Result}

In this section we will consider the Hamiltonian

$$
H=\sum_{j}\left(1-\sigma_{j}^{z}\right)-\varepsilon \sum_{\langle i j\rangle} \sigma_{i}^{x} \sigma_{j}^{x} .
$$

The sum over $\langle i j\rangle$ is over all nearest neighbor pairs. If we multiply this Hamiltonian by $\frac{1}{\varepsilon}$ we obtain the Hamiltonian of the quantum mechanical Ising model in a transverse magnetic field of magnitude $\frac{1}{\varepsilon}$. (Of course this overall factor of $\frac{1}{\varepsilon}$ is irrelevant since we are only interested in the ground state.) We will take the parameter $\varepsilon$ to be small, so the transverse field is large. For sufficiently small $\varepsilon$ this model has a unique infinite volume ground state [18]. We only consider the asymptotic behavior of the ground state expectation of $\sigma_{i}^{x} \sigma_{j}^{x}$ when the difference $i-j$ is along a lattice direction.

Theorem. Let $\hat{e}$ be a unit vector in one of the lattice directions. If $\varepsilon$ is sufficiently small then

$$
\left\langle\sigma_{0}^{x} \sigma_{n \hat{e}}^{x}\right\rangle \sim e^{-m|n|} /|n|^{d / 2}
$$

as $n \rightarrow \infty$. Here \langle\rangle denotes the unique infinite volume ground state. The two quantities are asymptotic in the sense that there exist constants $c_{1}>0$ and $c_{2}<\infty$ 
such that

$$
c_{1} e^{-m|n|} /|n|^{d / 2} \leqq\left\langle\sigma_{0}^{x} \sigma_{n \hat{e}}^{x}\right\rangle \leqq c_{2} e^{-m|n|} /|n|^{d / 2} .
$$

For $d=1$ this result was already known. The one dimensional model is closely related to the two-dimensional classical Ising model and has been solved exactly. Pfeuty [21] used results of Lieb et al. [16] and McCoy [19] to compute the expectation of $\sigma_{i}^{x} \sigma_{j}^{x}$ in the ground state for the one dimensional model. It has exponential decay with a power law correction of $|i-j|^{-1 / 2}$ when $\varepsilon$ is small.

The remainder of this section is devoted to the proof of this theorem. We begin by reviewing a well known geometric representation of expectations in this system [23]. Initially we work in a finite hypercube $\Lambda$ with periodic boundary conditions. We will develop a convergent expansion for our geometric representation, and the existence of the infinite volume limit will follow. A simple variant of the Trotter product formula implies

where

$$
\operatorname{Tr} e^{-\beta H}=\lim _{N \rightarrow \infty} \operatorname{Tr}\left\{\left[e^{-\frac{1}{N} H^{z}}\left(1-\frac{\varepsilon}{N} H^{x}\right)\right]^{N \beta}\right\}
$$

$$
H^{z}=\sum_{j \in \Lambda}\left(1-\sigma_{j}^{z}\right), \quad H^{x}=-\sum_{\langle i j\rangle \in \Lambda^{*}} \sigma_{i}^{x} \sigma_{j}^{x} .
$$

( $\Lambda^{*}$ denotes the set of nearest neighbor bonds in $\Lambda$.)

Inserting a sum over a complete basis between the factors in (2.2) we obtain

$$
\begin{aligned}
& \operatorname{Tr} e^{-\beta H}=\lim _{N \rightarrow \infty} \sum_{\psi_{1}, \ldots, \psi_{N \beta}}\left\langle\psi_{N \beta}\left|e^{-\frac{1}{N} H^{z}}\left(1-\frac{\varepsilon}{N} H^{x}\right)\right| \psi_{N \beta-1}\right\rangle \cdots \\
& \left\langle\psi_{2}\left|e^{-\frac{1}{N} H^{z}}\left(1-\frac{\varepsilon}{N} H^{x}\right)\right| \psi_{1}\right\rangle\left\langle\psi_{1}\left|e^{-\frac{1}{N} H^{z}}\left(1-\frac{\varepsilon}{N} H^{x}\right)\right| \psi_{N \beta}\right\rangle .
\end{aligned}
$$

Each $\psi_{t}$ is summed over the states in the orthonormal basis in which the $\sigma_{j}^{z}$ are diagonal. The matrix element $\left\langle\psi_{t+1}\left|e^{-\frac{1}{N} H^{z}}\left(1-\frac{\varepsilon}{N} H^{x}\right)\right| \psi_{t}\right\rangle$ factors into the product of $\left\langle\psi_{t+1}\left|e^{-\frac{1}{N} H^{z}}\right| \psi_{t+1}\right\rangle$ and $\left\langle\psi_{t+1}\left|1-\frac{\varepsilon}{N} H^{x}\right| \psi_{t}\right\rangle$. The second factor vanishes except in two cases. If $\psi_{t+1}$ is equal to $\psi_{t}$, then this matrix element is just 1 . If there is a bond $\langle i j\rangle$ such that $\psi_{t+1}=\sigma_{i}^{x} \sigma_{j}^{x} \psi_{t}$ then the matrix element is $\varepsilon / N$. The first factor simply equals $e^{-\frac{2 M}{N}}$, where $M$ is the number of -spins in the state $\psi_{t+1}$.

A choice of $\psi_{1}, \ldots, \psi_{N \beta}$ is equivalent to a classical Ising configuration on the $d+1$ dimensional lattice $\Lambda \times\{1,2, \ldots, N \beta\}$. We represent each configuration that occurs in the sum (2.3) by a set of bonds in this lattice. (The bonds will be bonds which join two lattice sites, not bonds in the dual lattice.) If site $i$ is - in the state $\psi_{t+1}$ then we include the bond $\langle(i, t),(i, t+1)\rangle$. We refer to such bonds as time bonds since they are parallel to the "time" direction. If $\psi_{t+1}=\sigma_{i}^{x} \sigma_{j}^{x} \psi_{t}$ for the bond $\langle i j\rangle$ then we include the bond $\langle(i, t),(j, t)\rangle$. These bonds will be called space bonds. There can be at most one space bond at any given time. A space bond implies that the spins at $i$ and $j$ flip at time $t$. Thus the spin at site $i$ will be - either before or after time $t$. The same remark applies to site $j$. Thus a space bond must have one time bond attached to each of its endpoints. Each of these time bonds can point forwards or backwards in the time direction. 
If the time bond $\langle(i, t),(i, t+1)\rangle$ is present, then the time bond $\langle(i, t+1),(i, t+2)\rangle$ must also be present unless there is a spin flip at time $t+1$. In other words a string of time bonds can only end by hitting a space bond. Each endpoint of a time bond can hit at most one space bond since there can be at most one space bond at any one time. These two restrictions imply that the collection of bonds corresponding to a term $\psi_{1}, \ldots, \psi_{N \beta}$ for which the matrix elements are all nonzero must form a set of disjoint loops. In the time direction these loops typically consist of a segment of $O(N)$ consecutive time bonds, which we refer to as a time segment. Furthermore, given any set of disjoint loops for which there is at most one space bond in each time slice, there is a choice of $\psi_{1}, \ldots, \psi_{N \beta}$ which is described by this set of loops. In other words there is a one to one correspondence between the terms in the sum in (2.3) and these sets of disjoint loops.

We associate the matrix elements with the loops in the following way. Each time bond implies a factor of $\exp (-2 / N)$, while each space bond implies a factor of $\varepsilon / N$. We define the weight $W(l)$ of a loop $l$ to be

$$
W(l)=\exp (-2 T(l) / N)\left[\frac{\varepsilon}{N}\right]^{S(l)}
$$

where $T(l)$ and $S(l)$ denote the number of time and space bonds respectively in the loop. Then we have shown

$$
\operatorname{Tr} e^{-\beta H}=\lim _{N \rightarrow \infty} \sum_{l_{1}, \ldots, l_{n}: d, *} \frac{1}{n !} W\left(l_{1}\right) \ldots W\left(l_{n}\right) .
$$

The sum is over all choices of disjoint loops. It implicitly includes a sum over $n$, the number of loops. The * on the sum stands for the global constraint that there can be at most one space bond at any given time, while the $d$ denotes the constraint that the loops must be disjoint. The factor of $1 / n$ ! arises because any collection of disjoint loops may be labelled in $n$ ! different ways.

The global constraint * makes it difficult to carry out an expansion for our system, but as we will show, it becomes irrelevant in the $N \rightarrow \infty$ limit. We should think of the lattice spacing in the time direction as being $1 / N$. Then one can see that in the $N \rightarrow \infty$ limit the probability that the constraint * is violated goes to 0 as $1 / N$. More precisely, we will prove that the above equation for $\operatorname{Tr} e^{-\beta H}$ is also valid if we omit the constraint *. Consider the bounds

$$
\begin{aligned}
& \operatorname{Tr}\left\{\left[\exp \left(-\frac{1}{N} H^{z}\right)\left(1-\frac{\varepsilon}{N} H^{x}\right)\right]^{N \beta}\right\} \\
& \quad \leqq \sum_{l_{1}, \ldots, l_{n}: d} \frac{1}{n !} W\left(l_{1}\right) \ldots W\left(l_{n}\right) \leqq \operatorname{Tr}\left\{\left[\exp \left(-\frac{1}{N} H^{z}\right) \exp \left(-\frac{\varepsilon}{N} H^{x}\right)\right]^{N \beta}\right\}
\end{aligned}
$$

The first inequality follows from the fact that all the weights are positive and so the sum (2.5) increases when we drop the constraint * on the terms allowed. To see why the second inequality is true, consider writing out the last trace in the form of.(2.3) and replacing $\exp \left(-\frac{\varepsilon}{N} H^{x}\right)$ by its power series. All the terms will be nonnegative and every term in the sum (2.5) will appear. Thus the result is an upper bound on (2.5). Finally we complete the proof by noting that as $N \rightarrow \infty$ the upper and lower bounds on (2.5) given in (2.6) both converge to $\operatorname{Tr} e^{-\beta H}$. Our proof that the constraint * is irrelevant took advantage of the nonnegativity of the weights $W(l)$. 
We should emphasize that a similar proof works for models in which the weights are not nonnegative. One simply bounds the difference between the sum with and without the constraint $*$ in terms of a model in which the off diagonal matrix elements of the Hamiltonian are replaced by their absolute value and then uses the above argument.

We now have a representation of the partition function as a gas of loops with a hard core condition, i.e., the loops cannot touch. If $\varepsilon$ is small the activities of these loops are small and we can hope to develop a convergent expansion for the logarithm of the partition function with the convergence uniform in $N$. The polymer expansion we must carry out is somewhat unusual in that the time direction is continuous. The condition for convergence of the polymer expansion given by Kotecky and Preiss [14] is ideally suited for this purpose. They proved that there is a convergent polymer expansion if one can find a constant $\varrho<1$ and a positive function $a(l)$ of the polymers $l$ (which are loops in our case) such that for each polymer $l^{\prime}$,

$$
\sum_{l: \ln l^{\prime} \neq \emptyset} W(l) e^{a(l)} \leqq \varrho a\left(l^{\prime}\right) .
$$

(The convergence of the polymer expansion when a similar condition holds can be proven by other methods, e.g., the methods in [6].) For a loop $l$ we define

$$
|l|=S(l)+T(l) / N \text {. }
$$

[Recall that $S(l)$ and $T(l)$ are the number of space and time bonds respectively in the loop.] Then we take $a(l)=|l|$. The definitions of $W(l)$ and $a(l)$ imply

where $\tilde{\varepsilon}=e \varepsilon$.

$$
W(l) e^{a(l)}=\left[\frac{\tilde{\varepsilon}}{N}\right]^{S(l)} \exp [-T(l) / N],
$$

We define the corners of a loop to be the sites $(i, t)$ such that both a time and a space bond hit $(i, t)$. Equivalently, the corners are the endpoints of the space bonds since two space bonds cannot share an endpoint. The set of corners of a loop $l$ will be denoted by $C(l)$. Note that $C(l)$ has $2 S(l)$ elements. The periodic boundary conditions in the time direction allow loops without any corners. For any site $i$ the set of bonds $\langle(i, t),(i, t+1)\rangle, t=1, \ldots, N \beta$, is a valid loop. Its weight is $e^{-2 \beta}$, so it will not contribute to the ground state.

If $l$ and $l^{\prime}$ overlap, then at least one of the following is true: $l^{\prime}$ contains a corner of $l, l$ contains a corner of $l^{\prime}$, or both of $l$ and $l^{\prime}$ have no corners. If the loop $l^{\prime}$ has corners then this observation implies

$$
\begin{aligned}
\sum_{l: \ln l^{\prime} \neq \emptyset} W(l) e^{a(l)} \leqq & \sum_{(k, t) \in l^{\prime}} \sum_{l:(k, t) \in C(l)} W(l) e^{a(l)} \\
& +\sum_{(k, t) \in C\left(l^{\prime}\right)} \sum_{l:(k, t) \in l} W(l) e^{a(l)} .
\end{aligned}
$$

The number of corners in the loop $l^{\prime}$ is equal to $2 S\left(l^{\prime}\right)$, while the number of sites $(k, t) \in l^{\prime}$ may be crudely bounded by $2 T\left(l^{\prime}\right)$. Since $2 S\left(l^{\prime}\right)+2 T\left(l^{\prime}\right) / N=2\left|l^{\prime}\right|$, it follows that (2.9) may be bounded by

$$
2\left|l^{\prime}\right| \max \left\{\sup _{(k, t)} N \sum_{l:(k, t) \in C(l)} W(l) e^{a(l)}, \sup _{(k, t)} \sum_{l:(k, t) \in l} W(l) e^{a(l)}\right\} .
$$

To bound the first of the two terms in this maximum we consider

$$
N \sum_{l:(k, t) \in C(l)}\left[\frac{\tilde{\varepsilon}}{N}\right]^{S(l)} \exp [-T(l) / N] .
$$


If we let $n=S(l)$, then the number of corners in $l$ is $2 n$. In addition to the $n$ space bonds the loop will contain $n$ time segments. (Recall that a time segment is a sequence of consecutive time bonds.) We can generate an arbitrary loop which has $(k, t)$ as a corner as follows. Starting at the corner $(k, t)$ we choose a time direction, i.e., forwards or backwards. This yields a factor of 2. Next we choose a length $t$ for the time segment. This can be anything from 1 to $N \beta$. The term $\exp [-T(l) / N]$ contains a factor of $\exp (-t / N)$ for each time segment, so the sum over this choice is bounded by

$$
\sum_{t=1}^{N \beta} \exp (-t / N) \leqq \sum_{t=1}^{\infty} \exp (-t / N) \leqq N \int_{0}^{\infty} e^{-t} d t=N .
$$

The time segment we have just chosen ends at some bonds $(k, s)$. Next we choose a space bond with one endpoint at $(k, s)$. This produces a factor of $2 d$. We repeat the above process a total of $n$ times. It is crucial to note, however, that in the last repetition, the choice of the length of the time segment is fixed by the constraint that $l$ must be a loop. Consequently there will be only $n-1$ factors of $N$ from (2.12). There are $n$ factors of $1 / N$ inside the sum in (2.11) and a factor of $N$ in front of the sum. Thus the factors of $N$ and $1 / N$ cancel exactly and the resulting bound is

$$
N \sum_{l:(k, t) \in C(l)}\left[\frac{\tilde{\varepsilon}}{N}\right]^{S(l)} \exp [-T(l) / N] \leqq \sum_{n=2}^{\infty}[4 d \tilde{\varepsilon}]^{n}=\frac{(4 d \tilde{\varepsilon})^{2}}{1-4 d \tilde{\varepsilon}} \text {. }
$$

To bound the second of the two terms in (2.10) we must consider

$$
\sum_{l:(k, t) \in l}\left[\frac{\tilde{\varepsilon}}{N}\right]^{S(l)} \exp [-T(l) / N] .
$$

The analysis is similar to the above with one important exception. We now generate the loop by starting from a site which is in the interior of one of the time segments rather than a corner. Thus there will be $n+1$ choices of lengths for the time segments. As before the last choice is fixed by the constraint that we have a loop. Thus we obtain $n$ factors of $N$ from (2.12) which exactly cancel the $n$ factors of $1 / N$ in (2.14). The resulting bound is again the right side of (2.13). We have shown that $(2.10)$ is bounded by

$$
2\left|l^{\prime}\right| \frac{(4 d \tilde{\varepsilon})^{2}}{1-4 d \tilde{\varepsilon}} .
$$

If the loop $l^{\prime}$ has no corners, the second term in the right side of (2.9) is not present but we must include a new term to include the possibility that $l$ is also a loop without corners. There is only one such $l$ which intersects $l$, namely $l$ itself. For this loop, $W(l) e^{a(l)}=e^{-\beta}$. Thus in this case

$$
\sum_{l: l \cap l^{\prime} \neq \emptyset} W(l) e^{a(l)} \leqq 2\left|l^{\prime}\right| \frac{(4 d \tilde{\varepsilon})^{2}}{1-4 d \tilde{\varepsilon}}+e^{-\beta} \leqq 2\left|l^{\prime}\right|\left[\frac{(4 d \tilde{\varepsilon})^{2}}{1-4 d \tilde{\varepsilon}}+e^{-\beta}\right] .
$$

We have assumed that $\beta \geqq 1 / 2$ so that the length $\left|l^{\prime}\right|$ of the cornerless loop $l^{\prime}$ is at least $1 / 2$.

Since $\left|l^{\prime}\right|=a\left(l^{\prime}\right)$, we have shown that (2.7) holds if

$$
\frac{(4 d \tilde{\varepsilon})^{2}}{1-4 d \tilde{\varepsilon}}+e^{-\beta}<1 / 2 .
$$


This inequality is true if $\varepsilon$ is sufficiently small and $\beta$ is sufficiently large.

Condition (2.7) implies there is a convergent expansion for the logarithm of the partition function. If we define a cluster to be a set of loops $l_{1}, \ldots, l_{n}$ such that $\bigcup_{i} l_{i}$ is
connected, then there is a function $\Phi^{T}(C)$ of the cluster $C$ such that

$$
Z=\operatorname{Tr} e^{-\beta H}=\exp \left[\sum_{c} \Phi^{T}(C)\right] .
$$

There are explicit formulae for $\Phi^{T}(C)$ (Eq. (2) of [14], or Theorem 3.1 of [6]), but we will mainly be concerned with bounds on it.

Having developed a convergent expansion for the partition function, we next consider the unnormalized correlation function $\operatorname{Tr}\left(e^{-\beta H} \sigma_{i}^{x} \sigma_{j}^{x}\right)$. The term $\sigma_{i}^{x} \sigma_{j}^{x}$ implies that the spins at sites $i$ and $j$ must each flip at time 0 . Geometrically this means there must be a time segment which either starts or ends at $i$ and a time segment which either starts or ends at $j$. The path which begins with the time segment at $i$ can only end at the time segment at $j$. Thus we have

$$
\operatorname{Tr}\left(e^{-\beta H} \sigma_{i}^{x} \sigma_{j}^{x}\right)=\sum_{l, l_{1}, \ldots, l_{n}: d} W(l) W\left(l_{1}\right), \ldots, W\left(l_{n}\right),
$$

where $l$ is summed over all paths from $(i, 0)$ to $(j, 0)$ and $l_{1}, \ldots, l_{n}$ are summed over all loops. As before the $d$ indicates that $l, l_{1}, \ldots, l_{n}$ must be pariwise disjoint. The weight $W(l)$ of the path $l$ is still given by (2.4).

We define

$$
Z(\backslash l)=\sum_{l_{1}, \ldots, l_{n}: d, l} W\left(l_{1}\right), \ldots, W\left(l_{n}\right),
$$

where the constraint $l$ means that the loops $l_{1}, \ldots, l_{n}$ cannot intersect $l$. This partition function also has a convergent expansion of the form (2.15). The only change is that only those clusters $C$ which are disjoint from $l$ appear in the sum. Thus (2.17) can be written as

$$
\sum_{l} W(l) Z(\backslash l)
$$

and so the normalized correlation function is

$$
\begin{aligned}
\left\langle\sigma_{i}^{x} \sigma_{j}^{x}\right\rangle & =\sum_{l} W(l) Z(\backslash l) / Z \\
& =\sum_{l} W(l) \exp \left[\sum_{C} \Phi^{T}(C)-\sum_{C: C \cap l=\emptyset} \Phi^{T}(C)\right] \\
& =\sum_{l} W(l) \exp \left[\sum_{c: C \cap l \neq \emptyset} \Phi^{T}(C)\right] .
\end{aligned}
$$

Before continuing the development of the expansion we should decide which terms in (2.18) are important and which ones are small. There is an important difference here between the classical and quantum cases. In the classical case, e.g. the Ising model in two dimensions at high temperature, the path $l$ will be a straight line most of the time with occasional excursions in the direction orthogonal to the line from $i$ to $j$ (see Fig. 1). These rare excursions may be thought of as excitations since each one carries a small factor (roughly equal to $\beta$ ). In the quantum case the continuous nature of the extra dimension implies that a typical walk will look like Fig. 2. There will be an excursion in the time direction at every site, and so these excursions cannot be treated as excitations. The excitations in the quantum case are when the walk contains space bonds which do not point in the direction of $i-j$. 


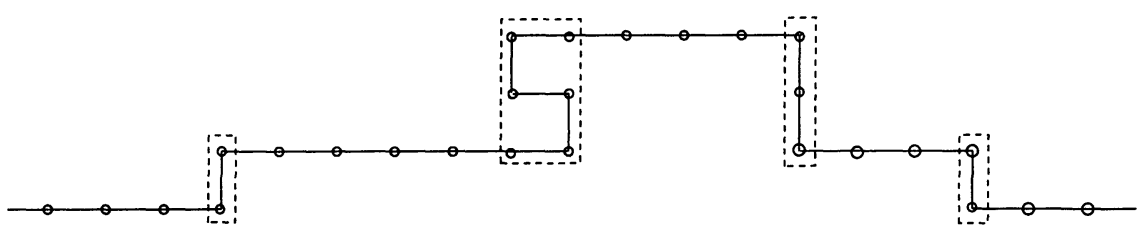

Fig. 1. A typical walk in the classical case in two dimensions. Most of the time the walk consists of horizontal bonds, but occasionally there is a vertical bond or the walk can even backtrack. These excitations are enclosed in dashed boxes

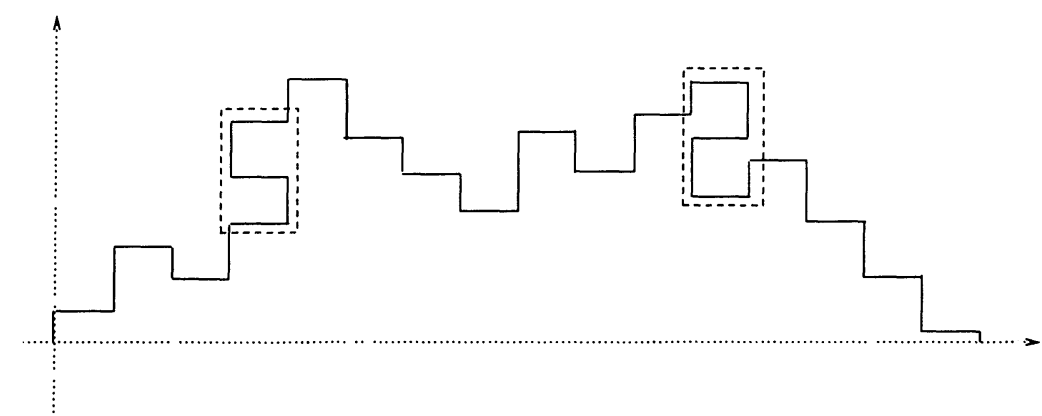

Fig. 2. A typical wal, in the quantum case in one space dimension (the horizontal axis). The vertical axis is the time axis. At every site the walk will have a bond of some length in the time direction. Excitations are when the walk backtracks (as shown in the dashed boxes) or sidetracks, i.e., contains a bond which points in one of the space directions other than the space direction shown. No examples of sidetracking are shown in the figure since they only occur when there are at least two space dimensions

We assume $\hat{e}$ is $e_{1}$, the unit vector in the 1-direction, and refer to the coordinate axis in the 1-direction as the 1-axis. If we think of the path $l$ as running from 0 to $n e_{1}$, then the bonds in $l$ have a direction. Most of the space bonds will point in the $e_{1}$ direction, but occasionally the path will backtrack or sidetrack and the space bonds will point in some other direction. We take the set of space bonds in $l$ which do not point in the $e_{1}$ direction and project this set onto the 1 -axis. The resulting set will consist of closed intervals which begin and end at lattice sites on the 1-axis and of isolated lattice sites on the 1-axis. We denote this set by $E(l)$ and call it the excitation set of $l$. To save some writing we will use the term $X$-interval to refer to a closed interval on the 1-axis whose endpoints are lattice sites including the degenerate case where the interval is a single lattice site. Note that $E(l)$ is a disjoint union of $X$-intervals.

Excitations can also arise from the clusters $C$ that appear in (2.18). The projection $\pi(C)$ of a cluster $C$ is the union of the projections of the loops in $C$ onto the 1-axis. Because $C$ is connected, $\pi(C)$ will be connected and thus $\pi(C)$ is a single $X$-interval. For an $X$-interval $X$ we define

Then

$$
K(X, l)=\sum_{C: C \cap l \neq \bar{\emptyset}, \pi(C)=X} \Phi^{T}(C) .
$$

$$
\sum_{C: C \cap l \neq \emptyset} \Phi^{T}(C)=\sum_{X} K(X, l),
$$

where the sum is over all $X$-intervals $X$ which intersect $\pi(l)$. 
We pause to bound $K(X, l)$. If $\pi(C)=X$ then the number of space bonds in the loops in $C$ must be at least as large as $|X|$, the length of $X$. Each of these space bonds carries a factor of $\varepsilon$, so we have a bound of the form

$$
|K(X, l)| \leqq e^{-M|X|}\left|l \cap \pi^{-1}(X)\right|,
$$

where we can take $M$ as large as we like by taking $\varepsilon$ sufficiently small. We now rewrite (2.18) as follows. First define

Then

$$
k(X, l)=\exp [K(X, l)]-1 .
$$

$$
\begin{aligned}
\sum_{l} W(l) \exp \left[\sum_{C: C \cap l \neq \emptyset} \Phi^{T}(C)\right] & =\sum_{l} W(l) \prod_{X}[1+k(X, l)] \\
& =\sum_{Y} \sum_{l: E(l)=Y} W(l) \prod_{X}[1+k(X, l)] \\
& =\sum_{Y} \sum_{X_{1}, \ldots, X_{n}} \sum_{l: E(l)=Y} W(l) k\left(X_{1}, l\right) \ldots k\left(X_{n}, l\right) .
\end{aligned}
$$

The $X_{1}, \ldots, X_{n}$ are summed over $X$-intervals which are distinct but not necessarily disjoint, i.e., $X_{i}$ and $X_{j}$ cannot be the same set but they may intersect. $Y$ is summed over subsets of the 1 -axis which are unions of disjoint $X$-intervals. Let $B=\bigcup_{i} X_{i} \cup Y$ and let $B=\bigcup_{i} B_{i}$ be the decomposition of $B$ into connected components. (Each $B_{i}$ will be an $X$-interval.)

Next we must divide the weight $W(l)$ among the connected components $B_{i}$. This weight $W(l)$ contains a factor of $\varepsilon / N$ for each space bond in $l$. The path $l$ begins and ends with time segments, so $l$ contains exactly one more time segment than space bonds. So rather than associating a factor of $\varepsilon / N$ with each space bond in $l$, we can associate a factor of $\varepsilon / N$ with each time segment and include an overall factor of $N / \varepsilon$. With this convention $W(l)$ simply consists of a factor of $\exp (-2 t / N) \varepsilon / N$ for each time segment of length $t$, and an overall factor of $N / \varepsilon$.

Next we consider sites on the 1-axis which are not in $B$. If $k$ is such a site, then the path $l$ contains only one time segment whose projection onto the 1-axis is the site $k$. The path $l$ must be going in the 1-direction when it enters $k$ and when it leaves $k$. Thus the behavior of the path $l$ at site $k$ is completely specified by a time $t_{k}$ (which can be negative or positive) which gives the length and direction of this time segment. We have a factor $\frac{\varepsilon}{N} \sum_{t_{k}}$ for each site not in $B$. We denote this factor by just $\varepsilon \int d t_{k}$. In addition $W(l)$ contains a factor of $\exp (-2 t / N)$ for each time segment of length $t$, and so a factor of $\exp \left(-2 t_{k} / N\right)$ for each site $k$ on the 1-axis which is not in $B$.

Now consider a component $B_{n}$ of $B$. By definition the sites just outside $B_{n}$ are not in $E(l)$. It follows that the path $l$ will contain exactly two space bonds which connect sites in $\pi^{-1}\left(B_{n}\right)$ to sites not in $\pi^{-1}\left(B_{n}\right)$. (If 0 or $n e_{1}$ is in $B_{n}$, then there will be one such bond.) We picture the site 0 as being to the left of the site $n e_{1}$ so that we may describe one of the space bonds as being on the left and one on the right. Let $t_{l}$ and $t_{r}$ be the times of the left and right such space bonds and define $s_{k}=t_{r}-t_{l}$. (If $0 \in B_{n}$ then $t_{l}=0$, and if $n e_{1} \in B_{n}$ then $t_{r}=0$.) Thus $s_{k}$ is the net change in the time produced by that part of $l$ in $\pi^{-1}\left(B_{n}\right)$ just as $t_{k}$ was the change in time associated with a site $k$ on the 1 -axis but not in $B$. Let $i_{r}$ and $i_{l}$ be the spatial coordinates of these two space bonds. Let $P$ be the $(d-1)$ dimensional hyperplane that is perpendicular to both the 1 -axis and the time axis. Let $j_{k}$ be the projection of $i_{r}-i_{l}$ 
onto $P$. So $j_{k}$ is the change in the second through $d^{\text {th }}$ spatial coordinates resulting from that part of $l$ associated with $B_{k}$. Considering where the walk $l$ begins and ends we see that the sum of all the times $t_{k}$ and $s_{k}$ must be zero and the sum of the lattice vectors $j_{k}$ must also be zero. (If $d=1$ then there is no plane $P$ and some of the following expressions simplify.)

Now consider the part of $W(l)$ that we should associate with $B_{n}$. Each time segment has a factor of $\exp (-2 t / N) \varepsilon / N$ where $t$ is the length of the time segment. Let $l_{n}$ denote the portion of $l$ which lies in $\pi^{-1}\left(B_{n}\right)$. The definition of the $B=\bigcup_{i} B_{i}$ decomposition implies $l_{n}$ will have only one connected component, and so $l_{n}$ is itself a path. [However, note that $E\left(l_{n}\right)$ need not be connected.] Let $W\left(l_{n}\right)$ be the product of the factors of $\exp (-2 t / N) \varepsilon / N$ in $W(l)$ coming from time segments whose projections onto the 1 -axis is a site in $B_{n}$. The weights $k\left(X_{j}, l\right)$ for $X_{j} \subset B_{n}$ do not depend on all of $l$, just $l_{n}$; in other words $k\left(X_{j}, l\right)=k\left(X_{j}, l_{n}\right)$ if $X_{j} \subset B_{n}$.

We now define for an $X$-interval $B$, a time $s$ and a lattice vector $j \in P$,

$$
W(B, s, j)=N \sum_{l, X_{1}, \ldots, X_{m}: B, s, j} k\left(X_{1}, l\right) \ldots k\left(X_{m}, l\right) W(l),
$$

where the sum is over all walks $l$ and $X$-intervals $X_{i}$ such that $\bigcup_{i} X_{i} \cup E(l)=B$. The sum over walks $l$ in this definition is subject to the following constraints. First suppose that neither of 0 or $n e_{1}$ is in $B$. Pick a site $k_{l}$ such that $\pi\left(k_{l}\right)$ is the left endpoint of $B$. Let $k_{r}$ be the site whose time coordinate equals the time coordinate of $k_{l}$ plus $s$ and whose spatial coordinate equals the spatial coordinate of $k_{l}$ plus $j$ plus $(|B|-1) e_{1}$, where $|B|$ is the number of lattice sites in the interval $B$. [So $|B|-1$ is the length of $B$ and $\pi\left(k_{r}\right)$ will be the right endpoint of $B$.] Then the walk $l$ in the above must begin at $k_{l}$ and end at $k_{r}$. Thus the walk must produce a time change of $s$ and a change in the second through $d^{\text {th }}$ spatial coordinates of $j$. This definition does not depend on the choice of the site $k_{l}$ because of the translation invariance. If $0 \in B$ then we take $k_{l}$ to be the site with spatial and time coordinates equal to 0 . If $n e_{1} \in B$ we replace $|B|-1$ in the above by the distance from the left endpoint of $B$ to $n e_{1}$. If both 0 and $n e_{1}$ are in $B$ we take $k_{l}=0$ and replace $|B|-1$ by $n$. Note that we have included a factor of $N$ in the definition of $W\left(B, S_{j}\right)$. This is a natural thing to do in view of the restriction that the change in the time resulting from $l$ is constrained to be a particular value $s$. Of course we will have to have a factor of $1 / N$ someplace else to cancel this $N$.

To bound $W(B, s, j)$ we use the bound on $K(X, l),(2.19)$ and the definition of $W(l)$. Note that since $K(X, l)$ is small, $k(X, l)$ is essentially equal to $K(X, l)$. It is straightforward to show that the sums in the definition of $W(B, s, j)$ converge using the techniques that we used to prove (2.7). We need to show more, namely, that $W(B, s, j)$ is small relative to the weight we get from walks which do not backtrack or sidetrack as they go through $B$. Recall that $|B|$ is the number of lattice sites in $B$. A walk which never backtracks or sidetracks will have $|B|$ factors of $\varepsilon$ associated with its passage through $B$ since it will have $|B|$ time segments associated with $B$, each of which carries a factor of $\varepsilon$. Likewise any term which contributes to $W(B, s, j)$ will have at least $|B|$ factors of $\varepsilon$. Each lattice site in $B$ must come from $\bigcup_{i} X_{i}$ or $E(l)$ and so will contribute an additional factor of $\varepsilon$. Let $|j|$ be the $l^{1}$ norm of $j$. Then $|j|$ is less than or equal to the number of bonds in $l$ which do not point in the positive $e_{1}$ direction. So the number of additional factors of $\varepsilon$ is at least $|j|$. Any walk which contributes to $W(B, s, j)$ will also contain a factor of $e^{-2|s|}$, so we can extract a factor of $e^{-|s|}$ and still control the sums in the definition of $W(B, s, j)$. The resulting 
bound is

$$
|W(B, s, j)| \leqq \varepsilon^{|B|} e^{-\mu|B|-|s|-\mu|j|},
$$

where $\mu$ can be made as large as we like by taking $\varepsilon$ sufficiently small.

Remembering the overall factor of $N / \varepsilon$, the correlation function is now

$$
\begin{aligned}
\left\langle\sigma_{i}^{x} \sigma_{j}^{x}\right\rangle= & \varepsilon^{-1} \sum_{B_{1}, \ldots, B_{m}} \int d s_{1} \ldots \int d s_{m} \sum_{j_{1}} \ldots \sum_{j_{m}} W\left(B_{1}, s_{1}, j_{1}\right) \ldots W\left(B_{m}, s_{m}, j_{m}\right) \\
& \times \prod_{i \notin B}\left(\varepsilon \int d t_{i} e^{-2\left|t_{i}\right|}\right) \delta\left(\sum_{k=1}^{m} s_{k}+\sum_{i \notin B} t_{i}\right) \delta^{(d-1)}\left(\sum_{k=1}^{m} j_{k}\right),
\end{aligned}
$$

where $B_{1}, \ldots, B_{m}$ are summed over disjoint $X$-intervals and $j_{1}, \ldots, j_{m}$ are summed over lattice vectors in $P$, the $(d-1)$ dimensional hyperplane orthogonal to the 1 -axis and the time axis. The set $B$ denotes $\bigcup_{i} B_{i}$. The function $\delta(t)$ is shorthand for the function that equals $N$ if $t=0$, and equals 0 otherwise. The function $\delta^{(d-1)}(j)$ is the function which equals 1 if $j=0$ and 0 otherwise $(j$ ranges over $P$ ). Recall that $\int d s_{i}$ is short for $\frac{1}{N} \sum_{s_{i}}$. This factor of $1 / N$ cancels the factor of $N$ that was included in the definition of $W(B, s, j)$. The global constraints are treated just as in the classical case [5], i.e.,

$$
\begin{gathered}
\delta(t)=\frac{1}{2 \pi} \int_{-N \pi}^{N \pi} d k \exp (i k t), \\
\delta^{(d-1)}(j)=\frac{1}{(2 \pi)^{d-1}} \int_{-\pi}^{\pi} d^{d-1} q \exp (i q \cdot j) .
\end{gathered}
$$

In the first integral $k$ runs from $-N \pi$ to $N \pi$. In the second integral $q$ runs over all points in $R^{d-1}$ with each component between $-\pi$ and $\pi$. Of course, the advantage of these representations is that the integrands factor over the polymers $B_{i}$.

For each site $i \notin B$ we have a factor of

$$
\varepsilon \int d t_{i} \exp \left(-2\left|t_{i}\right|+i k t_{i}\right)=\frac{\varepsilon}{1+k^{2} / 4} .
$$

The number of sites $i \notin B$ is $1+\mathrm{n}-\sum_{j}\left|B_{j}\right|$. Thus

$$
\left\langle\sigma_{i}^{x} \sigma_{j}^{x}\right\rangle=\frac{1}{\varepsilon(2 \pi)^{d}} \int_{-N \pi}^{N \pi} d k \int_{-\pi}^{\pi} d^{d-1} q Z(k, q)
$$

with

$$
\begin{aligned}
Z(k, q)= & \sum_{B_{1}, \ldots, B_{m}}\left(\frac{\varepsilon}{1+k^{2} / 4}\right)^{1+n-\sum_{j}\left|B_{j}\right|} \\
& \times \prod_{l}\left[\int d s_{l} \sum_{j_{l}} W\left(B_{l}, s_{l}, j_{l}\right) \exp \left(i k s_{l}+i q \cdot j_{l}\right)\right] .
\end{aligned}
$$

(The limits on the $k$ and $q$ integrals are as before.) We define

$$
\tilde{W}\left(B_{l}, k, q\right)=\left(\frac{\varepsilon}{1+k^{2} / 4}\right)^{-\left|B_{l}\right|} \int d s_{l} \sum_{j_{l}} W\left(B_{l}, s_{l}, j_{l}\right) \exp \left(i k s_{l}+i q \cdot j_{l}\right)
$$


so

$$
Z(k, q)=\left(\frac{\varepsilon}{1+k^{2} / 4}\right)^{n+1} \sum_{B_{1}, \ldots, B_{m}} \tilde{W}\left(B_{1}, k, q\right) \ldots \tilde{W}\left(B_{m}, k, q\right) .
$$

If $k$ is large then the factor of $\left(1+k^{2} / 4\right)^{|B|}$ in $\tilde{W}(B, k, q)$ is large. This makes it difficult to control an expansion for $\ln Z(k, q)$. Thus we first consider the case that $|k| \leqq K$, where $K$ is a constant to be chosen later. The bound $(2.20)$ on $W(B, s, j)$ and the definition of $\tilde{W}(B, k, q)$ imply

$$
|\tilde{W}(B, k, q)| \leqq\left(1+K^{2} / 4\right)^{|B|} e^{-\mu|B|} \sum_{j} e^{-\mu|j|} \int d s e^{-|s|} \leqq e^{-\mu^{\prime}|B|},
$$

where $\mu^{\prime}$ is essentially $\mu-2 \ln K$. The above estimate shows that $Z(k, q)$ is a polymer gas with small activities if $\mu \gg 2 \ln K$. Thus $\ln Z(k, q)$ has a convergent polymer expansion. We can write the result as

$$
Z(k, q)=\left(\frac{\varepsilon}{1+k^{2} / 4}\right)^{n+1} \exp [n f(k, q)+g(k, q, n)+h(k, q)] .
$$

$f(k, q)$ is the "free energy" per site between 0 and $n e_{1}$ and is independent of $n$. $g(k, q, n)$ comes from the terms in the expansion that reach all the way from 0 to $n e_{1}$. It is exponentially small in $n . h(k, q)$ comes from "boundary effects" and is independent of $n$.

If $|k|>K$ we do not attempt to prove the convergence of an expansion for $\ln Z(k, q)$. Instead we return to (2.22). We claim that $Z(k, q)$ for $|k|>K$ is exponentially small in $n$ relative to $Z(k, q)$ for $k$ near 0 . In (2.22) there is a factor of $\left(1+k^{2} / 4\right)^{-1}$ for each site not in $\bigcup_{j} B_{j}$. This is small if we take $K$ large. For sites in $\bigcup_{l} B_{l}$ we have smallness from the $W\left(B_{l}, s_{l}, j_{l}\right)$ factors. This proves the claim, so the correlation function $\left\langle\sigma_{i}^{x} \sigma_{j}^{x}\right\rangle$ is asymptotically given by (2.21) with the integral restricted to $|k| \leqq K$.

We now have

$$
\begin{aligned}
\left\langle\sigma_{i}^{x} \sigma_{j}^{x}\right\rangle \sim & \varepsilon^{n} \frac{1}{(2 \pi)^{d}} \int_{-K}^{K} d k \int_{-\pi}^{\pi} d^{d-1} q\left(1+k^{2} / 4\right)^{-(n+1)} \\
& \times \exp [n f(k, q)+g(k, q, n)+h(k, q)]
\end{aligned}
$$

with the usual limits on the integral over $q$. We pause now to deal with the $\beta \rightarrow \infty$ and $N \rightarrow \infty$ limits. (Recall that the $N \rightarrow \infty$ limit is the limit in the Trotter product formula.) For a fixed value of $N$ and $\beta$ we can take the infinite volume limit in our expansions, in particular in (2.23). The bounds which insure convergence of the expansions are uniform in $\beta$ and $N$. The usual application of the dominated convergence theorem shows that we can now take the $N \rightarrow \infty$ and $\beta \rightarrow \infty$ limits of the quantities $f(k, q), g(k, q, n)$, and $h(k, q)$.

Since $g(k, q, n)$ is exponentially small in $n$, the above is asymptotic to the same expression with $g(k, q, n)$ omitted. Thus we must consider the integral

$$
\varepsilon^{n} \frac{1}{(2 \pi)^{d}} \int_{-K}^{K} d k \int_{-\pi}^{\pi} d^{d-1} q \exp \left\{(n+1)\left[-\ln \left(1+k^{2} / 4\right)+f(k, q)\right]+h(k, q)\right\} .
$$

The function $f(k, q)$ has an expansion in powers of $\varepsilon$. The lowest order terms arise from walks $l$ which have one space bond pointing in a direction other than the positive $e_{1}$ direction. Such an excitation gives a $B$ that consists of a single site. The 
contribution to $\tilde{W}(B, k, q)$ from these excitations is

$$
\begin{aligned}
& \left(\frac{\varepsilon}{1+k^{2} / 4}\right)^{-1} \int_{-\infty}^{\infty} d t_{1} \int_{-\infty}^{\infty} d t_{2} \sum_{j:|j|=1} \varepsilon^{2} \exp \left[-2\left|t_{1}\right|-2\left|t_{2}\right|+i k\left(t_{1}+t_{2}\right)+i q \cdot j\right] \\
& =\frac{\varepsilon}{1+k^{2} / 4} \sum_{i=2}^{d} 2 \cos \left(q_{i}\right),
\end{aligned}
$$

where $q_{i}$ is the $i^{\text {th }}$ component of $q$. All the other terms that contribute to $f(k, q)$ are smaller by at least a factor of $\varepsilon$. It is easily seen that these terms are also even in $k$ and in each $q_{i}$. Thus $f(k, q)$ has its maximum at $k=0, q=0$ and the expansion of $f(k, q)$ about this maximum to second order in $k$ and $q$ and to first order in $\varepsilon$ is $-\varepsilon k^{2}(d-1) / 2-\varepsilon \sum_{i=2}^{d} q_{i}^{2}$. A standard steepest descent argument (see, e.g., [5]) now shows the above expression for the correlation function is asymptotic to $\varepsilon^{n} e^{e n} / n^{d / 2}$, where $\varrho$ is a constant that depends on $\varepsilon$. (One factor of $n^{-1 / 2}$ arises from the integral over $k$, and each integral over one of the $q_{i}$ 's gives a factor of $n^{-1 / 2}$.)

\section{Examples with Anomalous Decay}

Classical spin systems in which the truncated two-point function decays exponentially do not always have a $(d-1) / 2$ power law correction to this exponential decay. A well known example is the two dimensional Ising model at low temperatures for which the power law correction is $|i-j|^{-2}$ rather than $|i-j|^{-1 / 2}$ [11]. Even at high temperatures there are examples which do not have Ornstein-Zernike decay [22]. In this section we review some examples of quantum spin models where the power law correction is not $d / 2$.

In one dimension several quantum spin systems are exactly solvable. In two of these models the asymptotic behavior of the correlation functions in the ground state is understood well enough that the power law correction to the exponential decay is known. One of these models is the model studied in this paper as we discussed following the statement of the main theorem. The other model is the anisotropic $X Y$ model whose Hamiltonian is

$$
H=-\sum_{i}\left(\sigma_{i}^{x} \sigma_{i+1}^{x}+\alpha \sigma_{i}^{y} \sigma_{i+1}^{y}\right)
$$

with $0<\alpha<1$. Lieb et al. [16] found exact expressions for the two point functions in this model. McCoy [19] determined their asymptotic behavior. The correlation function $\left\langle\sigma_{0}^{z} \sigma_{n}^{z}\right\rangle$ vanishes when $n$ is even. For odd $n$ its decay is given by

$$
\left\langle\sigma_{0}^{z} \sigma_{2 n-1}^{z}\right\rangle \sim-\alpha^{2 n-1}(2 n-1)^{-2} .
$$

In the $y$-direction he found

$$
\left\langle\sigma_{0}^{y} \sigma_{n}^{y}\right\rangle \sim \alpha^{n} n^{-1} .
$$

In the $x$-direction there is long range order.

$$
\lim _{n \rightarrow \infty}\left\langle\sigma_{0}^{x} \sigma_{n}^{x}\right\rangle=\left(1-\alpha^{2}\right)^{1 / 2} .
$$

The decay of the truncated correlation function $\left\langle\sigma_{0}^{x} ; \sigma_{n}^{x}\right\rangle=\left\langle\sigma_{0}^{x} \sigma_{n}^{x}\right\rangle-\left(1-\alpha^{2}\right)^{1 / 2}$ depends on whether $n$ is even or odd,

$$
\begin{gathered}
\left\langle\sigma_{0}^{x} ; \sigma_{2 n}^{x}\right\rangle \sim \alpha^{2 n}(2 n)^{-1}, \\
\left\langle\sigma_{0}^{x} ; \sigma_{2 n-1}^{x}\right\rangle \sim \alpha^{2 n-1}(2 n-1)^{-2} .
\end{gathered}
$$


Note that none of these correlation functions have a power law correction of $n^{-d / 2}$ to their exponential decay.

There are many quantum spin systems in which the ground state has an exact description in terms of valence bonds, i.e., spin singlets. (References may be found in [3].) Most of these models have correlation functions that vanish when the distance is more than a few lattice spacings. We restrict our attention here to those models with non-vanishing correlation functions [2-4]. The simplest example of this type is the spin-1 chain with Hamiltonian $H=\sum_{i} S_{i} \cdot S_{i+1}+\frac{1}{3}\left(S_{i} \cdot S_{i+1}\right)^{2}$. The ground state of this Hamiltonian is known and the two-point function has been computed $[2,3]$. It equals a constant times $3^{-|i-j|}$. This is the decay one would expect in a classical one dimensional model. For the usual Heisenberg spin 1 chain, $H=\sum_{i} S_{i} \cdot S_{i+1}$, there is numerical evidence that the two point function decays as $\exp (-m|i-j|) /|i-j|^{1 / 2}[15,20]$.

Arovas et al. [4] showed that for a family of models, which includes the above spin 1 chain as well as models in any number of dimensions, there is a representation of the ground state in terms of a classical model in the same number of dimensions. In dimensions greater than one the two point function has not been computed exactly. For some models it has been shown to decay exponentially $[3,12]$, but there are no rigorous results on the power law correction to this exponential decay. It is expected that the power law correction will not be $d / 2$ [4].

Using a clever idea of Kirkwood and Thomas [13], Matsui [17] showed that given any finite range translationally invariant classical Hamiltonian for Ising spins and an inverse temperature $\beta$, there is a finite range translationally invariant quantum mechanical Hamiltonian in the same number of dimensions such that the $\sigma^{z}$ correlation functions in the ground state of the quantum model equal the correlation functions of the classical system at inverse temperature $\beta$. The simplest example of this construction is to construct a quantum mechanical spin chain whose ground state correlations equal those of the classical one dimensional Ising model at inverse temperature $\beta$ [17]. Let

$$
H=\sum_{i}-\left[\sigma_{i}^{x}+\frac{\varepsilon}{2} \sigma_{i-1}^{z} \sigma_{i}^{z}+\frac{\varepsilon}{2} \sigma_{i}^{z} \sigma_{i+1}^{z}+\alpha \sigma_{i-1}^{z} \sigma_{i+1}^{z}\right] .
$$

We let

$$
\psi(\sigma)=\exp \left[\beta \sum_{i} \sigma_{i} \sigma_{i+1}\right]
$$

A little calculation shows that $\psi(\sigma)$ is the ground state of $H$ if $\varepsilon=2 \cosh (2 \beta) \sinh (2 \beta)$ and $\alpha=-\sinh ^{2}(2 \beta)$. A little algebra shows that we can find a $\beta$ such that these equations hold if and only if $\varepsilon$ and $\alpha$ are related by

$$
(1-2 \alpha)^{2}-\varepsilon^{2}=1, \quad \alpha \leqq 0 .
$$

The correlation functions for the classical one dimensional Ising model, in particular the two point function, have pure exponential decay, so there is no power law correction to the decay of the two point function in the ground state of this quantum spin chain.

If $\alpha=0$, then the Hamiltonian (4.1) is unitarily equivalent to the Hamiltonian we studied in Sect. 2 . The unitary operator is $U=\prod_{i}\left(1+i \sigma_{i}^{y}\right) / \sqrt{2}$. We see that 
$U H U^{-1}$ equals the Hamiltonian of Sect. 2, while $U \sigma_{i}^{z} \sigma_{j}^{z} U^{-1}=\sigma_{i}^{x} \sigma_{j}^{x}$. In Sect. 2 we showed that $\left\langle\sigma_{i}^{x} \sigma_{j}^{x}\right\rangle$ has a $|i-j|^{-1 / 2}$ correction. If $\alpha$ is nonzero but small and $\varepsilon$ is small, then we can develop a convergent expansion for the expectation of this two point function in the ground state. If $\alpha$ and $\varepsilon$ are related by (4.2) then from the preceding paragraph we know the two point function will not have a power law correction. Where does the argument of Sect. 2. break down? When $\alpha$ and $\varepsilon$ are both small, (4.2) implies that $\alpha$ goes as $-\varepsilon^{2}$. The next nearest neighbor term $\sigma_{i}^{z} \sigma_{i+2}^{z}$ becomes $\sigma_{i}^{x} \sigma_{i+2}^{x}$ under the unitary transformation. Note that the action of this term in the Trotter product expansion is the same as that of $\sigma_{i}^{x} \sigma_{i+1}^{x}$ followed by $\sigma_{i+1}^{x} \sigma_{i+2}^{x}$. Both of these possibilities carry factors of $\varepsilon^{2}$ but they have opposite signs. Thus there will be cancellation and the analysis of Sect. 2 breaks down. It would be interesting to use the expansion method of Sect. 2 to show explicitly that there is pure exponential decay when (4.2) holds.

Acknowledgements. It is a pleasure to thank Jean Bricmont, Daniel Fisher, and Michael Fisher for helpful conversations and correspondence.

\section{References}

1. Albanese, C.: On the spectrum of the Heisenberg Hamiltonian. J. Stat. Phys. 55, 297 (1989)

2. Affleck, I., Kennedy, T., Lieb, E.H., Tasaki, H.: Rigorous results on valence-bond ground states in antiferromagnets. Phys. Rev. Lett. 59, 799 (1987)

3. Affleck, I., Kennedy, T., Lieb, E.H., Tasaki, H.: Valence-bond ground states in isotropic quantum antiferromagnets. Commun. Math. Phys. 115, 477 (1988)

4. Arovas, D.P., Auerback, A., Haldane, F.D.M.: Extended Heisenberg models of antiferromagnetism: analogies to the fractional quantum hall effect. Phys. Rev. Lett. 60, 531 (1988)

5. Bricmont, J., Fröhlich, J.: Statistical mechanical methods in particle structure analysis of lattice field theories. Part I: Nucl. Phys. B 251, 517 (1985); Part II: Commun. Math. Phys. 98, 553 (1985)

6. Brydges, D.: A short course on cluster expansions. In: Critical phenomena, random systems, gauge theories. Osterwalder, K., Stora, R. (eds.), Amsterdam: Elsevier 1986

7. Campanino, M., Klein, A., Fernando Perez, J.: Localization in the ground state of the Ising model with a random transverse field. Commun. Math. Phys. 135, 499-515 (1991)

8. Dobrushin, R.L., Kotecký, R., Shloshman, S.B.: The Wulf construction: global shape from local interaction (in preparation)

9. Gallavotti, G.: The phase separation line in the two-dimensional Ising model. Commun. Math. Phys. 27, 103 (1972)

10. Ginibre, J.: Existence of phase transitions for quantum lattice system. Commun. Math. Phys. 14, 205 (1969)

11. Hecht, R.: Correlation functions for the two-dimensional Ising model. Phys. Rev. 158, 557 (1967)

12. Kennedy, T., Lieb, E.H., Tasaki, H.: A two dimensional isotropic quantum antiferromagnet with unique disordered ground state. J. Stat. Phys. 53, 383 (1988)

13. Kirkwood, J.R., Thomas, L.E.: Expansions and phase transitions for the ground state of quantum Ising lattice systems. Commun. Math. Phys. 88, 569 (1983)

14. Kotecký, R., Preiss, D.: Cluster expansion for abstract polymer models. Commun. Math Phys. 103, 491 (1986)

15. Liang, S.: Monte Carlo calculations of the correlation functions for Heisenberg spin chains at $T=0$. Phys. Rev. Lett. 64, 1597 (1990)

16. Lieb, E., Schultz, T., Mattis, D.: Two soluble models of an antiferromagnetic chain. Ann. Phys. (N.Y.) 16, 407 (1961)

17. Matsui, T.: A link between quantum and classical Potts models. J. Stat. Phys. 59, 781 (1990)

18. Matsui, T.: Uniqueness of the translationally invariant ground state in quantum spin systems. Commun. Math. Phys. 126, 453 (1990) 
19. McCoy, B.M.: Spin correlation functions of the $X-Y$ model. Phys. Rev. 173, 531 (1968)

20. Nomura, K.: Spin correlation functions of the $S=1$ antiferromagnetic Heisenberg chain by the large-cluster-decomposition Monte Carlo method. Phys. Rev. B 40, 2421 (1989)

21. Pfeuty, P.: The one-dimensional Ising model with a transverse field. Ann. Phys. 57, 79 (1970)

22. Stephenson, J.: Ising model spin correlations on the triangular lattice. II. J. Math. Phys. 7, 1123 (1966)

23. Suzuki, M.: Relationship between $d$-dimensional quantal spin systems and $(d+1)$ dimensional Ising systems. Prog. Theor. Phys. 56, 1454 (1976)

24. Thomas, L.E., Yin, Z.: Low.temperature expansions for the Gibbs states of weakly interacting quantum Ising lattice systems. Commun. Math. Phys. 91, 405 (1983)

25. Thomas, L.E., Yin, Z.: Low temperature expansions for the Gibbs states of quantum Ising lattice systems. J. Math. Phys. 25, 3128 (1984)

Communicated by M.E. Fisher 
